\section{Estimation of Vitamin A}

THERE is some risk that the letter under the above heading in NaTuRE of January 25 may create the incorrect impression that certain decisions were rashly taken by the 1934 International Conference on Vitamin Standardisation and that those responsible for these decisions are taking no steps to resolve the difficulties which have arisen. That there could be no intention to give this impression is clear from the fact that, of the three signatories to that letter, one was himself a member of the International Conference and a second was present as expert in an advisory capacity, while both are members of the Vitamin A Sub-Committee (of the Accessory Food Factors Committee, appointed by the Medical Research Council and Lister Institute) which is now attempting to deal in a constructive manner with problems which have arisen in connexion with the International Vitamin A Standard.

Nevertheless, the letter of Messrs. Bacharach, Drummond and Morton may cause uneasiness in the mind of anyone interested in vitamin standardisation and unaware of the above facts. It therefore seems desirable to explain that the problems of vitamin standardisation are admittedly very difficult, and that the decisions taken by the International Conference were regarded as subject to the verdict of experience.

The doubt which has arisen regarding the accuracy of the factor 1600 , recommended for adoption by the International Conference, to relate the results of the spectroscopic examination for vitamin A with those obtained by biological tests, is well known, and this problem is at present the subject of active inquiry and experiment.

The possibility that the cod liver oil subsidiary standard may in some circumstances be unstable has also been reported, and information is awaited from the United States, where this material has been in use for some years, as officially issued by the Trustees of the United States Pharmacopœia.

It is well that the existence of these difficulties should be ventilated and that the views of anyone having experience of vitamin A standardisation be invited. It is, however, doubtful if the interests of international biological standardisation are furthered by destructive criticism on matters of temporary difficulty which are being investigated in this and other countries, and for which the knowledge at present available does not permit a better or a more permanent solution.

E. M. Hume.

(Secretary to the Vitamin A SubCommittee of the Accessory Food Factors Committee jointly appointed by the Lister Institute and the Medical Research Council.)

The Lister Institute,

Chelsea Bridge Road, London, S.W.1.

\section{Tissue Cultures Exposed to the Influence of a Mag- netic Field}

ExperIments have recently been performed in order to determine whether exposure to a magnetic field has any visible effect on normal cells cultivated in vitro. The tissue employed was obtained from the heart of chick embryos of eight to nine days incubation, and the cultures were prepared by the hanging drop technique in a medium of fowl plasma and saline extract of embryonic tissue. After the first sub. cultivation, the culture chosen for treatment was placed in a small incubator between the poles of a magnet, the glass slide bearing the culture lying in a vertical plane, midway between the poles. The field strength, as measured in the air gap between the poles (after removal of the incubator), was about 5,000 gauss.

The cultures were allowed to remain in the field for periods which varied between three and six hours, and were, in general, fixed and stained immediately after removal from the field; several specimens, however, were incubated for various periods before fixation. Cultures from the same batch, which had been incubated without exposure to the magnetic field, were used as controls, in each experiment.

The exposed cultures exhibited no visible abnormal. ities in the arrangement of chromosomes in the dividing cells. There was observed, however, a slight tendency to protoplasmic disintegration in some of the resting cells in the exposed cultures; but we were not able definitely to establish this in all the cultures.

Ruby Payne-Scott.

WM. H. Love.

Cancer Research Laboratory, University of Sydney. Dec. 10.

\section{Excretion of Amino Acids from the Root Nodules of Leguminous Plants}

AFTER having shown that amino acids appear in the medium in inoculated, but otherwise sterile, quartz sand cultures of leguminous plants, we assumed that these amino acids are excreted from the nodules. The experimental evidence then available did not, however, exclude the possibility that the excretion might be a function of the roots.

In order to establish definitely this point, we have now analysed the sand from sterile, uninoculated cultures of peas, grown on nitrate nitrogen. An aqueous extract of this sand contained only negligible traces of amino nitrogen, showing that no significant excretion had taken place. In our opinion, this result confirms the view that the excretion takes place from the nodules, as we have suggested in our earlier communications on the subject. We regard the excreted amino acids (aspartic acid, lycine) as the primary products of the nitrogen fixation which takes place in the root nodules, and not as decom. position products of proteins.

ARtTURI I. VIrTANEN.

T. LAINE.

SYNNÖVE v. HAUSEN.

Laboratory of the Foundation

for Chemical Research,

Helsingfors.

Jan. 10.

\section{Molecular Structure of Chitin in Plant Cell-Walls}

UP to the present time, no X-ray investigations have been carried out on the molecular structure of chitin as present in plant cell-walls. The cell-walls of the sporangiophores of the fungus Phycomyces proved to be excellent material for such studies. Distinct diagrams were obtained with this material, the fibre diagrams being identical with those obtained from chitin of animal origin (wing of Periplaneta). 